\title{
Analytic Hierarchy Process for assessing e-health technologies for elderly indoor mobility analysis
}

\author{
Elena Simona \\ Lohan \\ Dpt. of Electr. \& \\ Comm. Eng., \\ TUT, Finland \\ elena- \\ simona.lohan@ \\ tut.fi
}

\author{
Oana Cramariuc \\ Dep. of Physics, TUT, \\ Finland \\ Centrul IT pentru \\ Stiinta si Tehnologie, \\ Bucharest, Romania \\ oana.cramariuc@ \\ tut.fi
}

\author{
Łukasz Malicki \\ Knowledge Society \\ Association \\ ul. Grażyny 13/15 \\ lok. 221, 02-548 \\ Warszawa, Poland \\ Lukasz.Malicki@ \\ ssw.org.pl
}

\author{
Neja Samar \\ Brenčič \\ MKS Electr. \\ Syst./ IZRIIS \\ neja.samar- \\ brencic@ \\ izriis.si
}

\author{
Bogdan \\ Cramariuc \\ citst.ro
}

Centrul IT pentru Stiinta

si Tehnologie, Romania

bogdan.cramariuc@

\begin{abstract}
Accidental falls and reduced mobility are major risk factors in later life. Changes in a person's mobility patterns can be related with personal well-being and with the frequency of memory lapses and can be used as risk detectors of incipient neurodegenerative diseases. Thus, developing technologies for fall detection and indoor localization and novel methods for mobility pattern analysis is of utmost importance in e-health. Choosing the right technology is not only a matter of cost and performance, but also a matter of user acceptability and the perceived ease-of-use by the end user. In this paper, we employ an Analytic Hierarchy Process (AHP) to assess the best fit-to-purpose technology for fall detection and user mobility estimation. Our multi-criteria decision making process is based on the survey results collected from 153 elderly volunteers from $5 \mathrm{EU}$ countries and on 10 emerging ehealth technologies for fall detection and indoor mobility pattern estimation. Our analysis points out towards a Bluetooth Low Energy wearable solution as the most suitable solution.
\end{abstract}

\section{Categories and Subject Descriptors}

H.4 [Information interfaces and presentation]: Miscellaneous.

\section{General Terms}

Design, Human Factors.

\section{Keywords}

Analytic Hierarchy Process (AHP), elderly e-health care, fall detection, indoor mobility, user surveys.

\section{INTRODUCTION}

Falls are one of the principal sources of injuries and hospitalization for elderly [5]. Also, mobility is a good indicator of health status and changes in movement patterns may signal an increased risk of the onset of a Neurodegenerative Disease (ND) [13]. For example, moving back and forth in a repetitive way between the same places inside the house may be associated with mild memory losses, remaining in a sitting position for long periods of time may signal mild depression. There is accumulating support in the literature that one of the key factors in increasing the efficacy of an e-health tele-monitoring system is to incorporate in the e-health system the right technology for detecting abrupt falls and estimating the indoor location and mobility [3][11]. There are several emerging technologies to support the fall detection and mobility pattern analysis, as it will be discussed in detail in Section III. Each of these technologies has their advantages and drawbacks, and there are very few studies yet which analyze these technologies from the elderly users' point of view based on survey data. It is the goal of our paper to provide a new framework, based on user survey results, expert opinions and AHP for helping the e-health tele-monitoring system designer to choose the most suitable technology for fall detection and indoor positioning, as bases for mobility pattern analysis.

\section{ANALYZED POPULATION}

\subsection{Survey Methodology}

A survey on elderly preferences toward various technologies was conducted in five European countries: France (FR), Switzerland $(\mathrm{CH})$, Romania (RO), Poland (PL) and Slovenia (SL). It was based on structured interviews conducted by human operators using a questionnaire, specifically developed, in the local language. Most of the questions used during the survey were categorical, with fixed answers, selected from a number of example-situations. Few questions, such as when the respondents were asked to justify their preference toward a certain technology, were open type requiring a narrative response. The participants were selected based on the following two criteria: 1) above retirement age or with permanent disability; 2) living alone or households of maximum two inhabitants.

\subsection{Statistics on survey participants}

Table 1. Respondents characteristics based on surveys

\begin{tabular}{|l|l|l|l|l|l|l|}
\hline Country & RO & CH & FR & PL & SL & Total \\
\hline Number of respondents & 61 & 6 & 10 & 44 & 32 & $\mathbf{1 5 3}$ \\
\hline Av. number of flat rooms & 3.2 & 6.7 & 3.8 & 3.1 & 5.2 & $\mathbf{4 . 4}$ \\
\hline Average age [years] & 73. & 74. & 81. & 74. & 70. & $\mathbf{7 4 . 7}$ \\
& 1 & 3 & 7 & 0 & 3 & \\
\hline Most recurrent age & 65 & 72 & 86 & 65 & 66 & $\mathbf{6 5}$ \\
\hline \% of living alone & 27. & 50 & 60 & 27. & 25 & $\mathbf{3 8 . 0}$ \\
\hline \% female respondents & 60. & 50 & 60 & 45. & 75 & $\mathbf{5 8 . 2}$ \\
\hline
\end{tabular}




\begin{tabular}{|l|l|l|l|l|l|l|}
\hline & 6 & & & 4 & & \\
\hline $\begin{array}{l}\text { \% of respondents with } \\
\text { chronic condition(s) }\end{array}$ & $\begin{array}{l}78 . \\
7\end{array}$ & 66. & 80 & 59. & 53. & $\mathbf{6 7 . 5}$ \\
\hline
\end{tabular}

The main characteristics of the analyzed population are given in Table 1: the number of respondents per country, the average number of rooms in the respondent primary dwelling, the average and most recurrent respondent age (all being above 60 years old), and the percentages of people living alone, of female respondents and of respondents with at least one chronic health condition.

\section{USED TECHNOLOGIES}

Mobile health assistive technologies for remote monitoring of fall detection and mobility pattern analysis fall into two main categories: i) the wearable technologies, which requires that the users carries a tag, a sensor or a transmitter with her/him (e.g., embedded in clothes, as a bracelet or as a portable device such as the mobile phone), and ii) the device-free technologies, where certain wireless transceivers, tags and sensors are installed in the user home, but the user is not required to carry on any device.

\subsection{Wearable technologies}

Wearable technologies are the most widespread ones in the ehealth community. The user comfort is not the highest with these technologies, but they are typically more precise than the devicefree solutions.

RFID tags: Radio Frequency Identification (RFID) tags are becoming an attractive option for e-health applications, due to their low-cost, tracking and positioning capabilities [12]. Passive RFID tags can be embroidered in human clothes and they 'communicate' through backscattered power measurements with an in-room reader. RFID ranges are typically small (few m), allowing thus for proximity positioning. Recent RFID-based fall detectors have been studied in [5]. BLE tags: Bluetooth Low Energy is a Bluetooth version meant for low power applications. BLE-based solutions have been slightly investigated in the context of fall detection and indoor positioning [8]. Wearable BLE tags are already available. WiFi tags: WiFi technology is one of the most popular wireless technologies nowadays and is already heavily present around us: in houses, hospitals, universities, commuting halls, etc. Most portable wireless devices have nowadays a incorporated WiFi chipset and clothing embedded WiFi transceivers are becoming a reality. WiFi-based fall detectors were reported in [17] and WiFi-based positioning solutions are widespread [11]. Accelerometer-based wearable devices: they measure the human body acceleration along certain axes. 3D digital accelerometers are widely used in e-health monitoring [5]. Positioning estimation via an accelerometer typically requires few additional sensors, such as gyroscopes (measuring the direction change) and barometers (measuring the height change). UWB tags: Ultra Wide Band (UWB) technology is based on sending short time pulses over a very wide bandwidth, and achieving thus centimeter-level accuracy in positioning accuracy [11]. UWB is still a rather expensive technology and wearable solutions are still rather scarce [18].

\subsection{Device-free technologies}

The device-free or contact-less technologies do not require that users carry any device and thus they cannot be forgotten to be worn. Typically, such technologies offer a lower accuracy than their wearable counterpart, since most of them (with the exception of vision systems) are based on the human body influences on the signal strength fluctuations between the in-house tags and in- house receivers, when the person crosses the wave path. Some of them (e.g. vision systems) are quite privacy invasive, and thus have a low user acceptability, as our surveys showed [8].

RFID in-house systems: The same principles as for the RFID tags apply, but this time the tags are scattered all through the house, not carried on by the person. The human body changes the signal propagation characteristics and the readers can thus detect the human presence and movements [12]. BLE in-house systems: The technology is the same as with the case of the BLE tags apply, with the main difference that the tags are on fixed places inside the house (not carried on). The fall detection and positioning principles [19] are similar with the one from the RFID in-house system. WiFi in-house systems: Similarly with RFID and BLE in-house systems, the WiFi in-house systems are contact-less systems, where the Access point and the WiFi tags or transmitters are installed externally to the human wearable fabrics or pockets. The fall detection and positioning principles [12] are similar with the one from the RFID and BLE in-house system. UWB in-house systems: UWB in-house systems differ from UWB tags in the fact that they do not employ any wearable devices [4]. The fall detection and user position are based on the time of arrival of multipath reflections due to human body presence. Device-free UWB solutions are still scarce in the literature. Vision/camera-based systems: The vision systems are those systems requiring at least one surveillance camera in users' homes. The surveillance cameras capture continuously the images of the users and analyze their movement patterns and behavioral changes based on vision navigation and pattern matching techniques [6]. Other technologies: Tactile or smart floors can also offer a device-free solution for user status monitoring indoors. The estimation accuracy depends on the density of the pressure sensing nodes, and the information can be sent to the central server through the WiFi network [2]. Tactile floors are however too expensive and disruptive technologies for the use in elderly homes, and thus they are not included in our analysis. Acoustic and ultrasound solutions have also been investigated in the context of elderly automatic monitoring of activities and indoor positioning [2][12].

\section{ANALYTIC HIERARCHY PROCESS}

Analytic Hierarchy Process (AHP) belongs to the category of Multi Criteria Decision Making (MCDM) processes, which derive ratio scales from paired comparisons between criteria and factor [1][2]. AHP can help the decision makers to choose between various options by taking into account both quantitative and qualitative factors. The priority weights can be gathered based on user surveys and expert opinions, as done here.

\subsection{Problem decomposition}

A block diagram of the AHP decision tree is shown in Figure 1: a decision regarding the suitability of a certain technology can be reached by taking into account several criteria and by dividing the problem into an hierarchical process: the first-level hierarchy shows the criteria according to which a decision is reached, and the second-level hierarchy shows the options (or technologies) to be analyzed. Each level has a certain priority factor or weight associated to it, here denoted by $w_{i}$ (first level) and $v_{i j}$ (second level), $i=1,2, \ldots, j=1,2, \ldots$ According to previous [8] and the current user surveys [3][22], the most important factors to evaluate the quality of a e-health technological solution for fall detection and indoor positioning/mobility analysis are: 
1. The system cost: this includes the component costs, and the installation and maintenance costs.

2. The technology acceptability by the end user refers to the subjective appreciation of users whether a certain technology would be acceptable or not to be installed in their homes (e.g., camera based solutions tends to be less acceptable than non-visual sensor-based solutions due to privacy concerns).

3. The ease of use of the technology: this refers to how much input, effort and technological knowledge is required from the user's side in order to use a certain technology.

4. The accuracy of the solution provided by the technology: here, it refers to positioning accuracy, which is also directly related to the accuracy of deriving viable mobility patterns.

5. The false alarm rates: in here, it refers to the rate of detecting and reporting false falls to the caregivers.

\subsection{AHP equations}

Once the main decision criteria are chosen (Figure 1), pair-wise comparison matrices at each hierarchy level can be built.

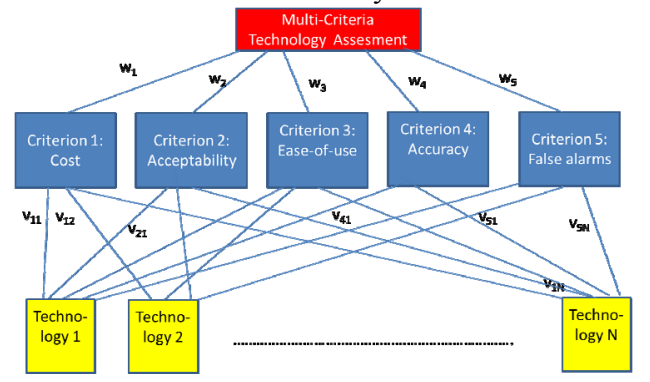

Figure 1. Problem decomposition via AHP.

A pair-wise comparison matrix $A_{h}=\left\{a_{i j}\right\}_{i, j=1, N_{h}}$ for hierarchy level $h$ (here, $h=1,2$ ) is built as given in eq. (1), where the $a_{i j}$ weights tells us how many times a column criterion (criterion $i$ ) is more important than a row criterion (criterion $j$ ). The lower diagonal elements of $A_{h}$ are obviously the inverses of the upper diagonal elements of $A_{h}$.

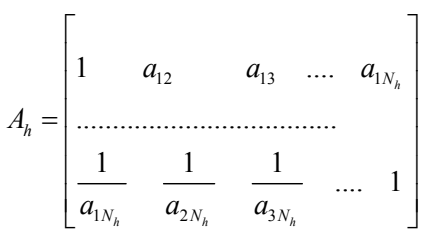

The normalized version of $A_{h}$ is the matrix $M_{h}$ with elements:

$$
M_{h}=\left\{m_{i j}\right\}_{i, j=1, N} \text { with } m_{i j}=\frac{a_{i j}}{\sum_{i=1}^{N} a_{i j}}
$$

The so-called priority vector $V_{h} \in R^{1 \times N}$ is obtained via:

$V_{h}=\frac{\operatorname{sum}\left(A_{h}{ }^{T}\right)}{N_{h}}, h=1,2$,

where $A_{h}{ }^{T}$ stands for the transpose of matrix $A_{h}$. The elements of the priority vector are the weights at each hierarchical level: for the first hierarchical level: $V_{1}=\left\{w_{i}\right\}_{i=1, N}$ and $V_{i 2}=\left\{v_{i j}\right\}_{j=1, N}$, $i=1, N$ for the second hierarchical level of Figure 1.
A decision about the best technology according to the multicriteria of levels 1 and 2 in of Figure 1 is taken by computing the final priority levels $t_{i}$ of each technology and sorting the technology according to its priority level:

$t_{i}=\sum_{j=1}^{N_{1}} w_{i} v_{i j}, i=1, N_{2}$

\subsection{AHP pair-wise comparison matrices}

Based on our survey results with the survey data in Table 1, on literature searches and on expert opinions based on discussion between authors, the following level 1 pair-wise comparison matrix has been obtained (Table 2). For example, this tells us that the Cost is 4 times more important than the accuracy from the user's point of view and the ease of use is twice more important than the false alarm rate.

Table 2. Level-1 pairwise comparison matrix

\begin{tabular}{|l|l|l|l|l|l|}
\hline & Cost & Acceptability & $\begin{array}{l}\text { Ease of } \\
\text { use }\end{array}$ & Accuracy & $\begin{array}{l}\text { False } \\
\text { alarm }\end{array}$ \\
\hline Cost & 1 & $1 / 2$ & 2 & 4 & 4 \\
\hline Acceptability & 2 & 1 & 4 & 10 & 5 \\
\hline Ease of use & $1 / 2$ & $1 / 4$ & 1 & 2 & 2 \\
\hline Accuracy & $1 / 4$ & $1 / 10$ & $1 / 2$ & 1 & $1 / 2$ \\
\hline False alarms & $1 / 4$ & $1 / 5$ & $1 / 2$ & 2 & 1 \\
\hline
\end{tabular}

The consistency ratio of Table 2 matrix is $2 \%$, which is much below the $10 \%$ consistency, showing thus a very good consistency of the data. The five level 2 pairwise comparison matrices are shown in our supplementary material of [8] due to lack of space. In order to build those, we used an average dwelling size of 4.4 rooms (as based on surveys, Table 1) and we assumed that the wearable solutions need one tag/user and the device-free solutions need 4 tags/room. These assumptions are based on literature studies and authors' knowledge on the technological needs in indoor positioning. The in-house RFID and UWB systems also require one reader per room (due to line of sight requirements), while in-house BLE and WiFi solutions work with one receiver per house. The vision-based system was also assumed to require one surveillance camera per room. The level 2 priority weights according to each criterion and to each technology are summarized in Table 3 which also shows which technology is the best among others with respect to a certain criterion. The letters stand for: A) Wearable BLE tag + in-house receiver (rx) ; B) Wearable RFID tag + in-house readers; C) Wearable WiFi tag + in-house receiver; D) Wearable UWB tag + in-house rx; E) Wearable accelerometer tag + in house rx; F) Inhouse BLE system (user is device free); G) In-house RFID system; H) In-house WiFi system; I) In-house UWB system; J) Vision-based system/ video cameras. Higher priority means better technology. It also shows which are the drawbacks and advantages of a certain technology with respect to a certain criterion. For example, technology A (wearable BLE tag) is the most cost effective technology, while technology $\mathrm{J}$ (vision-based system) is the easiest to be used among the 10 considered ones.

Table 3. Level-2 priority weights $v_{i j}[\%]$

\begin{tabular}{|l|l|l|l|l|l|l|l|l|l|l|}
\hline Techn & A & B & C & D & E & F & G & H & I & J \\
\hline Cost & 28.2 & 4.6 & 23.5 & 1.9 & 22.3 & 4.3 & 2.9 & 5.9 & 1.4 & 4.8 \\
\hline Accep & 13.0 & 13.0 & 13.0 & 13.0 & 13.0 & 9.8 & 6.2 & 9.8 & 6.2 & 3.1 \\
\hline $\begin{array}{l}\text { Ease } \\
\text { of use }\end{array}$ & 6.2 & 8.3 & 2.0 & 4.2 & 2.1 & 14.6 & 14.6 & 14.6 & 12.5 & 20.8 \\
\hline Accur & 1.5 & 1.5 & 0.75 & 30.1 & 0.6 & 1.5 & 3.0 & 0.8 & 30.1 & 30.1 \\
\hline $\begin{array}{l}\text { False } \\
\text { alarm } \\
\text { s }\end{array}$ & 1.0 & 1.5 & 1.0 & 29.4 & 5.9 & 0.7 & 1.0 & 0.7 & 29.4 & 29.4 \\
\hline
\end{tabular}




\section{SUITABILITY RANKING}

The 2-level AHP analysis based on the pairwise comparison tables and eq. (4) gives the suitability ranking. The technologies are ranked from the most suitable (rank 1) to the least suitable, by taking into account the user preferences and the 5 optimization criteria of Table 2 . The gaining technology is a solution based on wearable BLE tags and an in-house BLE receiver, followed closely by wearable accelerometer and wearable WiFi solutions, while the least suitable technology is an in-house (contact-less) RFID system, no doubt due to high cost, low accuracy and low comfort when installed in the house. The suitability according to AHP, given as percentages, is as follows: rank 1: Wearable BLE tag + in-house rx $(14.41 \%)$; rank 2: Wearable accelerometer tag + in house rx (12.71\%); rank 3: Wearable WiFi tag + in-house rx (12.64\%); rank 4: Wearable UWB tag + in-house receiver (11.27\%); rank 5: Vision System $(9.40 \%)$; rank 6: In-house UWB (8.93\%); Wearable RFID tag + in-house readers $(8.70 \%)$; rank 7: In-house WiFi system (8.22\%); rank 8: In-house BLE system (7.84\%); rank 9: In-house RFID system (5.84\%).

\section{CONCLUSIONS}

Choosing the right technology to support e-health solutions via fall detection and user mobility patterns analysis is a challenging problem. The aim of our paper has been to identify which of the existing technologies for indoor positioning, fall detection and mobility pattern analysis can satisfy most of the requirements of elderly with respect to acceptability, ease of use and cost, by taking into account also the performance indicators (i.e., accuracy and false rates). An AHP analysis was used based on user survey data collected in 5 EU countries. The result of our analysis show that the most suitable technology among the 10 most promising ones in the field of fall detection and indoor mobility is a technology based on a wearable BLE tag and additional fixed inhouse receiver. Our analysis also shows that wearable technologies are preferable to the device-free technologies, mostly because their better performance and lower associated costs. Another observation is that none of these technologies has a significantly higher priority than the others (the highest priority level is $14.4 \%$, only slightly higher than the $10 \%$ likelihood, which is the likelihood of randomly selecting one of these 10 technologies), which points out towards the fact that stand-alone solutions may be unable to address all optimality criteria and more advanced hybrid architectures are needed to be created.

\section{ACKNOWLEDGMENTS}

This work was supported by the following projects: Academy of Finland (projects 250266 and 283076), EU AAL NITICS, Mobile@Old, PN-II-PT-PCCA-2013-4-2241 No 315/2014.

\section{REFERENCES}

[1] Aflaki, S. Meratnia, N., Baratchi, M., Havinga, P. (2013). Evaluation of incentives for body area network-based healthcare systems. IEEE Intelligent Sensors, Sensor Net. and Inf. Proc., 515-520, Apr.

[2] Basiri, A., Peltola, P., Silva, P., Lohan, E., Moore, T., Hill C (2015). Indoor Positioning Technology Assessment using Analytic Hierarchy Process for Pedestrian Navigation Services. IEEE ICL GNSS, Sweden, Jun.

[3] Igual, R., Medrano C, Plaza, I. (2013). Challenges, issues and trends in fall detection systems. BioMedical Engineering OnLine, 12:66.
[4] Irahhauten, Z., Nikookar, M., Klepper, M. (2012). A joint TOA/DOA technique for 2D/3D UWB localization in indoor multipath environment. IEEE Int. Conf. on Comm., 4499-4503, Jun.

[5] Karantonis, D., Narayanan, M., Mathie, M., Lovell, N., Celler, B (2006). Implementation of a real-time human movement classifier using a triaxial accelerometer for ambulatory monitoring. IEEE Trans. On Inf. Techn. in Biomedicine, vol. 10(1), 156 - 167.

[6] Kawaji, H, Hatada, K., Yamasaki, T., Aizawa, K. (2010). Imagebased Indoor Positioning System: Fast Image Matching using Omnidirectional Panoramic Images. ACM MPVA, Oct, Italy.

[7] Koblasz, A. (2010). Using RFID to prevent or detect falls, wandering, bed egress and medication errors. US patent US 7714728 $B 2$, May.

[8] Lohan, E., Cramariuc, O., Malicki, L., Samar, N., Cramariuc,B. (2015) [Online supplementary material] http://goo.gl/GuklMB

[9] Lohan, E.S., Rusu-Casandra, A., Cramariuc, O., Marghescu, I., Cramariuc, B. (2011). End-User Attitudes towards Location-Based Services and Future Mobile Wireless Devices: The Students' Perspective. MDPI Information, vol. 2(3), 426-454.

[10] Mager, B., Patwari, N., Bocca, M. (2013). Fall Detection Using RF Sensor Networks. IEEE PIMRC, 3472-3476.

[11] Mautz, R. (2012). Indoor positioning technologies. Habilitation thesis, ETH Zurich, Feb.

[12] Moyer, V.A. (2012). Prevention of Falls in Community-Dwelling Older Adults: U.S. Preventive Services Task Force Recommendation Statement. Annals of Int. Medicine, vol. 157(3), 197-204.

[13] Ni Scanaill, C., Carew, S., Barralon, P., Noury, N., Lyons, D., Lyons, G.M. (2006). A Review of Approaches to Mobility Telemonitoring of the Elderly in their Living Environment. Annals of Biomedical Engineering, vol. 34(4), 547-563.

[14] Osterweil, J. (2009). Method and apparatus for body position monitor and fall detect ion using radar. US patent US7567200 B1, July.

[15] Saaty, T.L. (2008). Decision making with the analytic hierarchy process. Int. J. Services Sciences, vol. 1(1), 83-98.

[16] Schwarzmeier, A., Weigel, R., Fischer, G., Kissinger, D. (2014). A low power fall detection and activity monitoring system for nursing facilities and hospitals. IEEE Conf. on Biomedical Wireless Technologies, Networks, and Sensing Systems, 28-30, Jan.

[17] Tarng, W., Lin, C.H., Liou, H.H. (2012). Applications of wireless sensor networks in fall detection for senior people. Int. Journal of Computer Science \& Information Technology, vol 4(4), 79-95.

[18] Teng, X.F., Zhang, Y.T., Poon, C.Y., Bonato, P. (2008). Wearable Medical Systems for p-Health. IEEE Rev. in Biomedical eng., vol.1, $62-74$

[19] Vuegen, L., van den Broeck, B., Karsmakers, P., Van Hamme, H., Vanrumste, B. (2003). Automatic monitoring of activities of daily living based on real-life acoustic sensor data: A preliminary study. 4th workshop on speech and language processing for assistive technologies, 113-118, France, Aug.

[20] Xhafa, F., Moore, P., Tadros, G. (2015). Advanced Technological Solutions for E-Health and Dementia Patient Monitoring. IGI Global, 1-389. Web. 8 May. 2015. doi:10.4018/978-1-4666-7481-3

[21] Yanying, G., Lo, A., Niemegeers, I. (2009). A survey of indoor positioning systems for wireless personal networks., IEEE Commun. Surv. Tutorials, vol. 11, 13-32.

[22] Zielinska, I., Malicki, L., Samar Brencic, N., Consoli, A., Ayadi, J., Gilardi, L., Cramariuc, O., Stanciu, D., Didi, T., Smidtas, S. D2.1. The results of the multi-national survey. NITICS project public deliverables, http://nitics.eclexys.com/node/6. 